\title{
Piezoresistive Sensor for Strain Measurement on Turbine Blade with Wireless Telemetry Data Acquisition
}

\author{
P. Kulha ${ }^{1}$, M. Husak ${ }^{1}$ \\ ${ }^{1}$ Department of microelectronics, Czech Technical University, \\ Technicka 2, 16627 Prague, \\ Czech Republic \\ Phone number: +420 224352 793, e-mail: kulhap@fel.cvut.cz, husak@fel.cvut.cz
}

\begin{abstract}
In the paper, strain-gauge sensor design and its application for wireless measurement of blade deformations and strains are presented. The strain sensor was designed using FEM (Finite Element Modeling) and uses piezoresistive strain sensitive boron doped nanocrystalline layers or insulated silicon layers (SOI). Nanocrystalline Diamond (NCD) is a very promising material for fabrication of harsh-environment devices because of its unique mechanical and electrical properties. The prospective of using diamond is not only in sensors (MEMS) but in RF and power electronic as well. Selection of method for data transfer is the key problem in rotating mechanisms. Traditional approach is using the slip ring, but it is not suitable for multisensor applications due to the requirement of one slip ring per sensing element. Telemetry can be related to transfer a data over any media, but is typically applied on wireless communications. A basic telemetry system consists of a measuring element or sensor, a device for sending the transmission, a receiver, and an output controller which records and displays data.
\end{abstract}

\section{Key words}

Piezoresistivity, nanocrystalline diamond, strain measurement, telemetry, wireless data acquisition.

\section{Introduction}

Piezoresistivity is one of the widely utilized physical phenomena in different kinds of sensor devices. The basic function of the strain gauge is based on transforming the strain in certain direction as to change its electric resistance.

It allows measuring plenty of non-electrical quantities such as deformation, bending, force, acceleration etc.

A transducer obtains the monitored signal and converts the value into an electrical signal ready for transmission. However, telemetry applications which support large numbers of measured channels are too costly and are impractical when used with separate transmission channels for each measured quantity. Measurements are formatted or multiplexed and transmitted. This paper describes a low cost, low power, embedded wireless transmission system for data acquisition, signal processing using microcontroller and saving of measured data on a PC host.

\section{Sensor Design and Fabrication}

\section{A. 3D Modeling}

The software package Coventor has been used for design of mechanical and thermal characteristics of the structure. The tools enable design, modeling and successive modification of designed MEMS structures. Generally, the software package enables: drawing of 2D layout and its editing, simulation of production process, generation of 3D model from 2D masks, generation of network by the method of finite elements, solution of mechanical, electrostatic, thermal, piezoresistive, induction, optical, and further simulations.

Two different strain gauge topologies were designed and simulated. The first with single resistors or half bridges (of length from $500 \mu \mathrm{m}$ to $2000 \mu \mathrm{m}$ and of width from 25 $\mu \mathrm{m}$ to $200 \mu \mathrm{m}$,) on single side fixed cantilever beam mechanical transducer (Fig.1), the second with four resistors forming the Wheatstone bridge on membrane mechanical transducer.

\section{B. Technology of Fabrication of Sensors Used}

Two different piezoresistive thin layers were utilized in fabricated samples. The first was widely used P-type silicon layer on insulating $\mathrm{SiO}_{2}$ which enhances the hightemperature performance (SOI). The second was novel technology utilizing boron doped nanocrystalline thin film layers (NCD). 


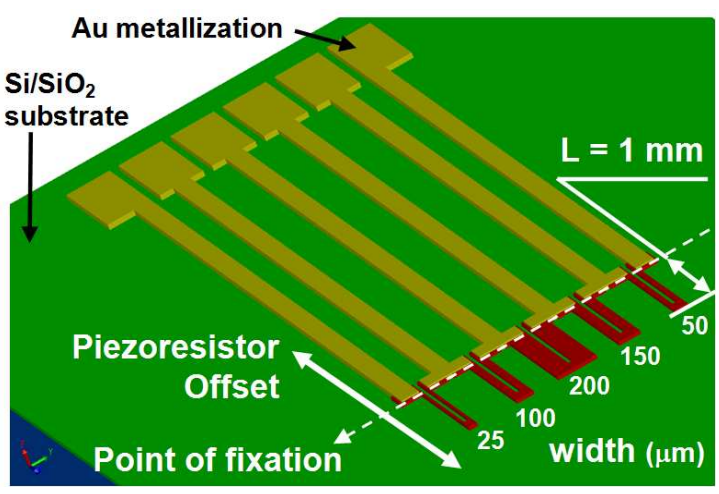

Fig. 1. 3D model of single resistor piezoresistive structures based on nanocrystalline thin film layer

\section{1) SOI fabrication process}

Flexible photosensitive films with designed topology mounted on carrier glass were used as photolithographic masks. Single resistors, meanders and bridge structures were designed. Standard photolithography and wet etching of contact and piezoresistive layers were utilized to prepare the samples. The wafers were coated with UVsensitive photoresist and lithographically patterned by exposing the photoresist to UV light through the first level mask, and then developed. The photoresist in exposed areas was removed, leaving behind a patterned photoresist mask for etching. Wet etching was used to etch the silicon down to the oxide layer. CP4 acid solution was used to etch silicon. After etching, the photoresist was chemically stripped. The $\mathrm{AlCuSi}$ metal layer was sputtered on the wafer, then patterned (through the second level mask) and etched to make electrical interconnections (Fig. 2).

\section{2) NCD fabrication process}

The sensor structures uses piezoresistive boron doped diamond thin films which were deposited on $\mathrm{SiO}_{2} / \mathrm{Si}_{3} \mathrm{~N}_{4} / \mathrm{Si}$ substrates of size $8 \times 25 \mathrm{~mm} 2$ (Fig.2.). After the deposition, all samples were lithographically processed and the piezoresistive structures were formed by reactive ion etching in an $\mathrm{O}_{2} / \mathrm{CF}_{4}$ gas mixture. The thickness of the diamond film was $250 \div 300 \mathrm{~nm}$. Finally, Ti and Au thin films were evaporated on piezoresistive structures to form metal contacts of $90 \mathrm{~nm}$ in thickness (Fig. 3).

Detailed information regarding fabrication of piezoresistive layer and its characterization can be found here [1],[2].

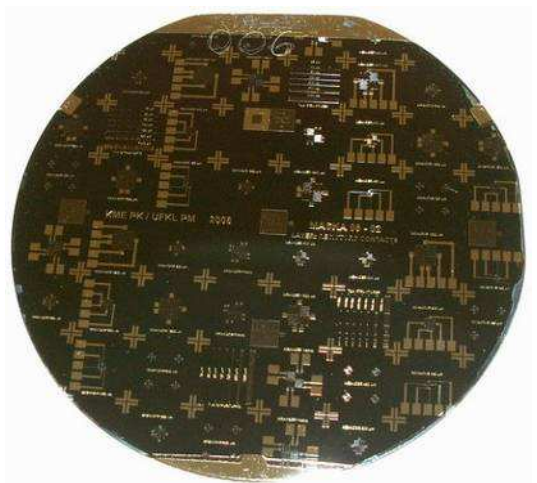

Fig. 2 Fabricated 4" wafer with SOI sensor structures

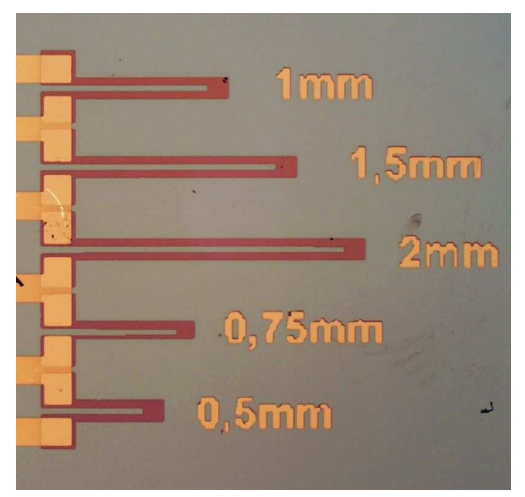

Fig. 3. Cantilever beam structures with detail of NCD piezoresistors

\section{Telemetry System for Data Acquisition}

Generally, short distance telemetry is a system to capture sensor output signals and transmit data over a short distance, of up to a few centimeters, to a receiver. The system comprises of a sensor and signal conditioning, which may be combined as a smart sensor, an encoding system and transmitter, an antenna and a power source [3]. The receiver is connected to a host, usually in the form of a PC, which performs analysis and displays results. As encoding occurs before transmission, the sensors can be either analogue or digital.

Telemetry can be used in small confined spaces or harsh environments that were previously inaccessible. With the use of micro and nano technology, the systems can be modular, compact and take advantage of the low power and low voltage requirements of these technologies. Embedded systems or integrated 'systems on a chip' are low cost and low weight, eliminate cables and use wireless radio technology (RF, Bluetooth, ZigBee transfer) [4]. Power sources available include inductive coupling, MEMS or rechargeable vaiteries, and are determined by application for suitability.

\section{A. Bridge Configuration of the Sensor}

Sensing piezoresistors are connected to the Wheatstone bridge. This configuration provides basic temperature compensation and increases sensitivity since all piezoresistors are strained (by tensile or compressive stress diagonally, see Fig. 3.)

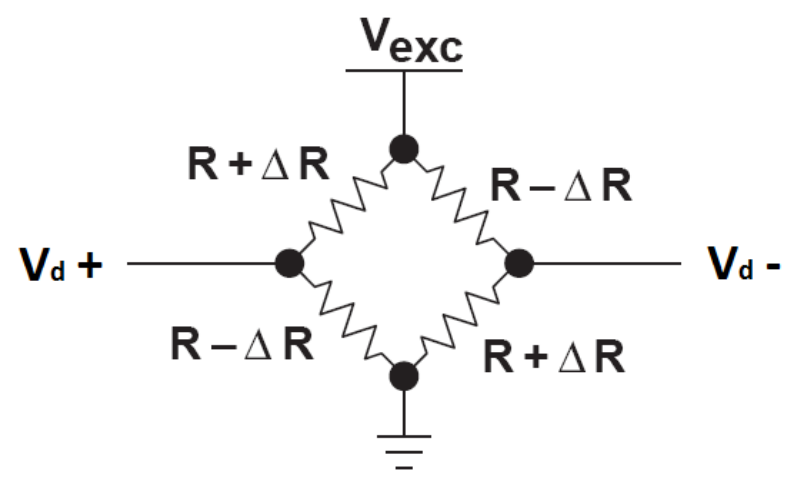

Fig. 4. Piezoresistive Wheatstone bridge 


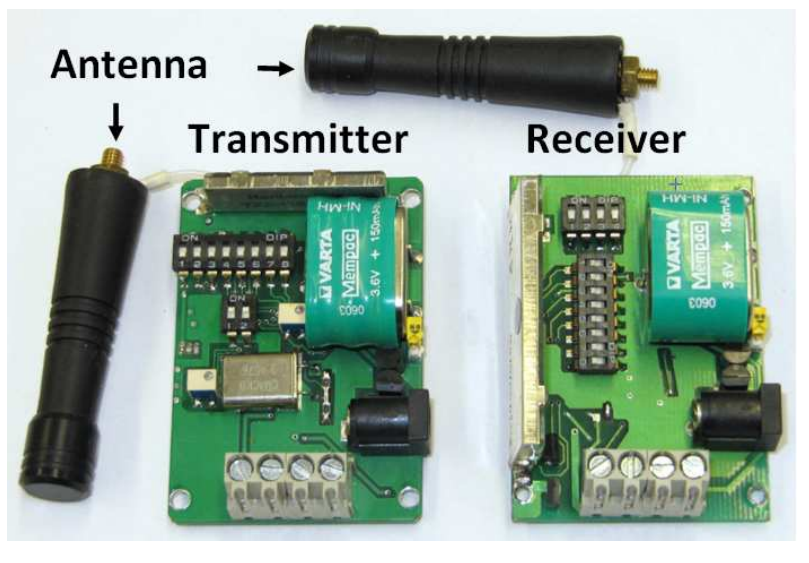

Fig. 5. RF transmitter and receiver modules for wireless linking

\section{B. Transmitter}

The transmitter used easily interfaces with any resistorbased or strain gage-based sensor and converts the sampled analog signal to digital code. This code is sent to the receiver via RF transmission. There, the original analog signal is reconstructed and a scaled output signal is produced.

The built-in radio transmitter and receiver circuits operate on $433 \mathrm{MHz}$ and are type approved, allowing license-free use in EU(see Fig. 5.). The operating ranges depend on an antenna used. With a fixed, compact, 1/4 wave helical antenna on both transmitter and receiver units enables lineof-sight operating ranges up to several meters [5].

The compact design incorporates miniature microprocessor-based circuitry, which provides 16-bit analog-to-digital conversion and digital encoding for the radio transmission.

The transmitter interfaces directly with a Wheatstone bridge sensor. An excitation voltage of $3.3 \mathrm{Vdc}$ powers the sensor. Bridge sensitivities from $\pm 2 \mathrm{mV} / \mathrm{V}$ to $\pm 1000 \mathrm{mV} / \mathrm{V}$ are available for maximum sampling sensitivity.

\section{Receiver}

The receiver reconstructs the signal from the transmitter, and produces a scaled output of 0 to $\pm 2.5 \mathrm{Vdc}$. An 8-way DIP switch sets the transmitter and receiver address codes. The 8-bit address code prevents interference from adjacent sets, and up to 256 codes are available [5].

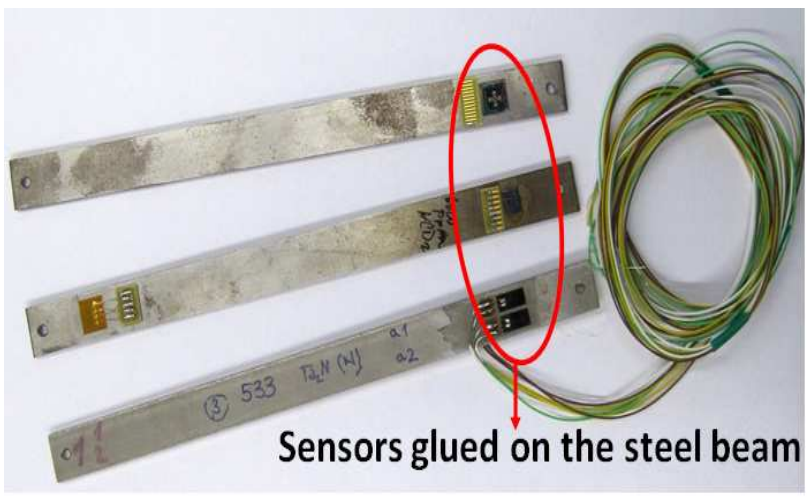

Fig. 6. Cantilever beams (blades) with glued sensors

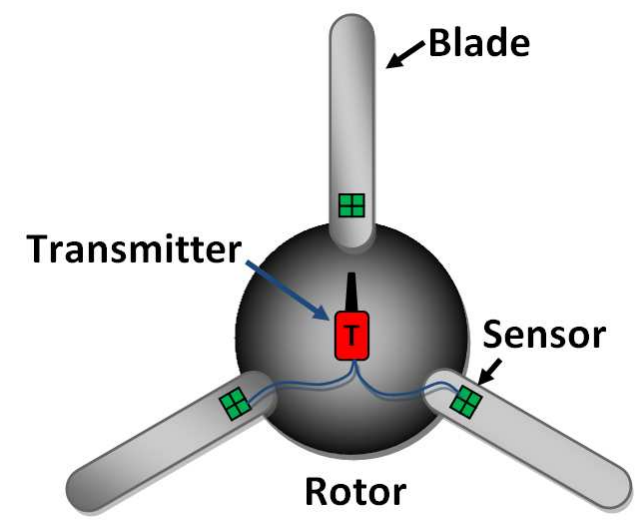

Fig. 7. Simplified drawing of rotor with indication of sensor position on blade

\section{Sensor Characterization and Testing of the Telemetry System}

\section{A. Sensor Parameters and Characterization Setup}

The fabricated chip was glued to the steel beam $(15 \mathrm{~mm} \mathrm{x}$ $200 \mathrm{~mm}$ ) using Hottinger EP310 (or TT300) epoxy resin. The sensitive elements are glued close to the point of fixation of the beam, where maximum deformation stresses occur. The beam was considered as a substitute of the turbine blade on real rotor (see Fig. 6.). Ball bonding technique was used to connect contact pads from sensing elements to a gold plated terminal board.

The extensive study of sensor parameters e.g. deformation sensitivity, edge and contact resistances, temperature dependences gauge factor, bridge output voltage in both static and dynamic loading was performed. Major sensor parameters are summarized in Table I.

Table I - Properties of NCD and SOI piezoresistors

\begin{tabular}{lll}
\hline Parameter & NCD & SOI \\
\hline Resistivity $(\Omega * \mathrm{~cm})$ & 0.01 & 0.02 \\
Sheet resistance $(\Omega)$ & 270 & 75 \\
Gauge Factor $(-)$ & $8 \pm 0.5$ & $45 \pm 5$ \\
TCR $(1 / \mathrm{K})$ & $-4.4 \times 10^{-4}$ & $12.2 \times 10^{-4}$ \\
Measured range & $25 \div 250{ }^{\circ} \mathrm{C}$ & $25 \div 200{ }^{\circ} \mathrm{C}$ \\
\hline
\end{tabular}

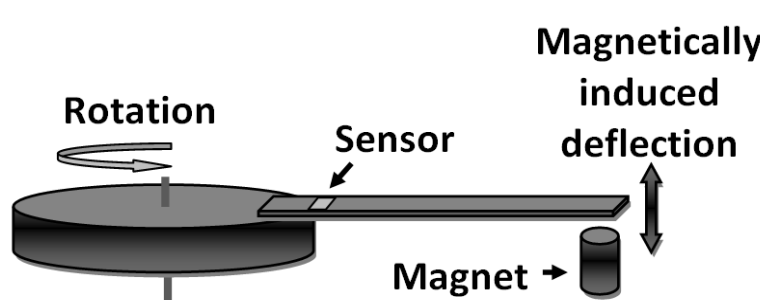

Fig. 8. Simplified model of a turbine rotor with magnetically induced deflection of the blade for testing of the system 


\section{B. Model of a Turbine Rotor and Characterization Setup}

The measurement and telemetry system was tested in laboratory conditions, since application inside real power generator or on wind generator was impossible at this stage of development. Realized measurement setup consisted of simplified model of turbine rotor with steel beam blades and sensor elements (Fig. 7.), transmitter mounted in the center of the rotor, receiver, cDAQ acquisition system (National Instruments) and Labview virtual instrument for evaluation of measured data.

The deflection of steel cantilever beams is done by electromagnet located under the free end of beam (Fig. 8). The gap between electromagnet and beam is approximately $8 \div 10 \mathrm{~mm}$. Pull-in force is driven by amount of current flowing to electromagnet. Maximum static deflection of the beam induced by magnetic force is up to $5 \mathrm{~mm}$.

The speed of the rotor is $\sim 1000 \mathrm{rpm}$. The beam bending (while passing over the electromagnet) is detected as a change in bridge voltage. Fig. 9. depicts result from the measurement (transmitter output) in the case of moving rotor. There are two waveforms, the first belongs to the beam with SOI piezoresistors, the second belong to the NCD piezoresistors. The force impulse causes the highest peak, and then short eigen oscillations occur. The ratio between peaks corresponds with the ratio of deformation sensitivity (Gauge Factor) of sensor used.

\section{Discussion}

Both SOI and NCD piezoresistors are suitable for detection of deformation and stresses on bended blade with sufficient sensitivity. SOI sensors exhibits better sensitivity, but NCD piezoresistors are more suitable for harsh environment applications (mainly higher temperatures, e.g. inside power turbine generators). Disadvantage of NCD piezoresistors is that the fabrication technology is demanding and costly.

The functionality of the wireless telemetry link is good, the disadvantage is that one unit can receive signal just from one transmitter at the same time.

For future work is considered better miniaturization of the transmitter, using other transfer protocol (Zig-Bee), adaptation of the receiver for multiplexing several transmitters, and application on real rotating sytem.

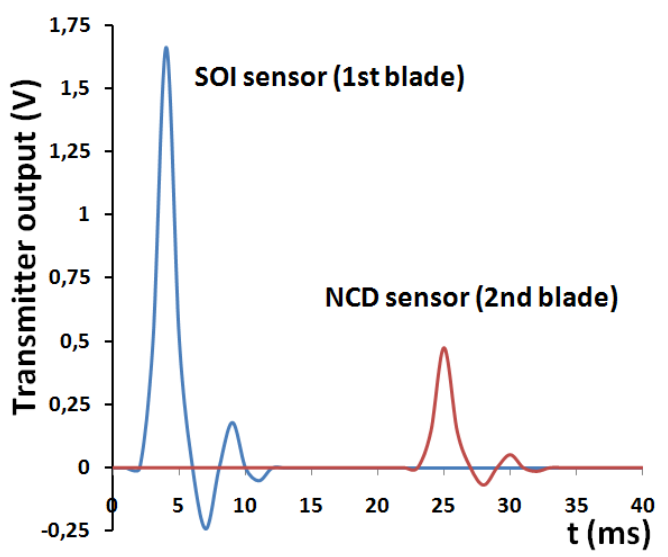

Fig. 9. Transmitter output waveform measured on rotor in motion

\section{Conclusions}

The system developed can be used in applications where is impossible or impractical to use conventional hardwire connections. The system uses novel strain sensor based on nanocrystalline (NCD) layers suitable for harsh environment applications and also SOI sensors used for comparative study. Modern methods (FEM simulations) were used in the sensor design. The applications of the whole system (sensor, signal conditioner, wireless link and data acquisition) cover full diagnostic measurement on rotational devices such as rotational parts of a wind turbine generator or inside electrical power generators. The system is modular and can be easily adapted to measure other electrical or nonelectrical quantities such as pressure and temperature.

\section{Acknowledgement}

This research has been supported by the Czech Science Foundation project No. 102/09/1601 "Micro- and nanosensor structures and systems with embedded intelligence" and partially by the research program No. MSM6840770015 "Research of Methods and Systems for Measurement of Physical Quantities and Measured Data Processing" of the CTU in Prague.

\section{References}

[1] P. Kulha, A.Kromka, O. Babchenko, et al. "Nanocrystalline Diamond Piezoresistive Sensor", Vacuum, 2009, vol. 84, no. 1 , p. 53-56. ISSN 0042-207X.

[2] P. Kulha, A. Boura, M. Husak, "Design and Fabrication of Piezoresistive Strain-Gauges for Harsh Environment Applications", in Proc. ICREPQ'10, 2010, pp. 510

[3] R. Puers, "Linking sensors with telemetry: impact on the system design", in Sensors and Actuators A: Physical Volume 52, Issues 1-3, March-April 1996, Pages 169-174

[4] N. Van Helleputte, J.M. Tomasik, W. Galjan, at al., "A flexible system-on-chip (SoC) for biomedical signal acquisition and processing", Sensors and Actuators A: Physical, 2008, Volume 142, Issue 1, Pages 361-368

[5] RT400 transmitter and receiver datasheet, www.omega.com 